\title{
Clinical features and outcomes of influenza by virus type/subtype/ lineage in pediatric patients
}

\author{
Seung Beom Han ${ }^{1,2}$, Jung-Woo Rhim ${ }^{1}$, Jin Han Kang ${ }^{1,2}$, Kyung-Yil Lee ${ }^{1}$ \\ ${ }^{1}$ Department of Pediatrics, College of Medicine, The Catholic University of Korea, Seoul, Korea; ${ }^{2}$ The Vaccine Bio Research Institute, College of \\ Medicine, The Catholic University of Korea, Seoul, Korea \\ Contributions: (I) Conception and design: JH Kang, KY Lee; (II) Administrative support: JH Kang, KY Lee; (IV) Collection and assembly of data: SB \\ Han, KY Lee, JW Rhim; (V) Data analysis and interpretation: All authors; (VI) Manuscript writing: All authors; (VII) Final approval of manuscript: \\ All authors. \\ Correspondence to: Jung-Woo Rhim, MD, PhD. Department of Pediatrics, Daejeon St. Mary's Hospital, College of Medicine, The Catholic University \\ of Korea, 64 Daeheung-ro, Jung-gu, Daejeon 34943, Korea. Email: jwrhim@catholic.ac.kr.
}

Background: Recently, four influenza viruses are circulating worldwide: A(H1N1)pdm09, A(H3N2), B/ Victoria, and B/Yamagata. However, information on the clinical differences among pediatric patients infected with four recently circulating influenza viruses is sparse.

Methods: Medical records of pediatric patients ( $<20$ years of age) diagnosed with influenza between the 2014-2015 and 2018-2019 influenza seasons were retrospectively reviewed. Clinical features were compared between (I) patients infected with influenza A (FluA) and influenza B (FluB) viruses, (II) patients infected with FluA when $\mathrm{A}(\mathrm{H} 1 \mathrm{~N} 1)$ pdm09 and $\mathrm{A}(\mathrm{H} 3 \mathrm{~N} 2)$ circulated dominantly, and (III) patients infected with FluB when B/Victoria and B/Yamagata circulated dominantly.

Results: A total of 1,588 patients infected with FluA and 964 patients infected with FluB were included in this study. Patients infected with FluB were older $(\mathrm{P}<0.001)$ and more likely to report sore throat $(\mathrm{P}=0.002)$ than those infected with FluA. Otherwise, there were no significant differences in the clinical symptoms, diagnoses, and outcomes between patients infected with FluA and FluB. Overall, clinical features of influenza patients were similar regardless of the dominantly circulated subtype and lineage of the virus. In children aged $\leq 2$ years, patients infected with FluB were more like to experience lower respiratory tract infection $(\mathrm{P}=0.034)$ and hospitalization $(\mathrm{P}=0.001)$ than those infected with FluA.

Conclusions: There were no significant clinical differences among pediatric patients infected with four recently circulating influenza viruses, except that FluB infection tended to be more severe than FluA infection in children aged $\leq 2$ years.

Keywords: Influenza; influenza A virus; influenza B virus; children

Submitted Jul 16, 2020. Accepted for publication Nov 30, 2020.

doi: $10.21037 / \mathrm{tp}-20-196$

View this article at: http://dx.doi.org/10.21037/tp-20-196

\section{Introduction}

Among influenza A, B, C, and D viruses, influenza A and $B$ viruses cause seasonal influenza in humans (1). The differences in epidemiological and clinical features and outcomes by influenza virus type, subtype, and lineage can influence strategies for influenza vaccination and treatment. Children, especially those younger than 5 years of age, have higher rates of hospitalization and mortality than adults (2,3), and school-age children are reported to drive the spread of influenza in the community during influenza epidemics (4). Therefore, information on clinical and epidemiological characteristics of pediatric patients with influenza should be investigated for the appropriate control and prevention of influenza in the general population as well as in school settings. Previous studies have shown similar symptoms, signs, and severity between patients infected 
with influenza A and B viruses; however, inconsistent results were found according to the type and subtype of circulating influenza viruses, characteristics of the included patients (e.g., age, hospitalized state, the presence of chronic underlying diseases), diagnostic methods for influenza, and antiviral therapy (5). Following the 2009 pandemic caused by influenza A(H1N1)pdm09 virus, A(H1N1)pdm09 and $\mathrm{A}(\mathrm{H} 3 \mathrm{~N} 2)$ have been the dominant influenza $\mathrm{A}$ virus circulating worldwide (1). The epidemiological and clinical features of influenza A virus infection have likely changed in the several years since $A(H 1 N 1) p d m 09$ became a seasonal influenza strain. For influenza B viruses, two lineages (Victoria and Yamagata lineages) have been circulating worldwide since the 1980s (6). Although B/Yamagata and B/ Victoria lineages have caused seasonal epidemics alternately or concurrently since the 2000s (6), there are only a few reports on the clinical differences between pediatric patients infected with B/Yamagata and B/Victoria $(7,8)$. In one study, children infected with B/Victoria were significantly older than those infected with B/Yamagata (7). Children infected with B/Yamagata experienced more frequent lower respiratory tract infection (LRI) than those infected with B/Victoria, whereas, the duration of hospitalization was significantly longer in children infected with B/Victoria than in those infected with B/Yamagata (7). However, the enrolled children of the previous study were not vaccinated against influenza at all, and the study was performed in the early 2000s. Considering that trivalent influenza vaccines, which have been administered for several decades, include antigens from both $\mathrm{A}(\mathrm{H} 1 \mathrm{~N} 1) \mathrm{pdm} 09$ and $\mathrm{A}(\mathrm{H} 3 \mathrm{~N} 2)$, but only one of B/Yamagata and B/Victoria, the difference in vaccine-acquired immunity against influenza $A$ and $B$ viruses may cause some differences in epidemiological and clinical features between influenza $\mathrm{A}$ and $\mathrm{B}$ virus infections and between B/Yamagata and B/Victoria infections.

In this study, clinical features and outcomes of pediatric patients diagnosed with influenza between the 2014-2015 and 2018-2019 influenza seasons were investigated, and were compared according to influenza virus type, subtype, and lineage. We present the following article in accordance with the STROBE reporting checklist (available at http:// dx.doi.org/10.21037/tp-20-196).

\section{Methods}

\section{Subject and data collection}

Pediatric patients (<20 years of age) in whom influenza was diagnosed at the Daejeon St. Mary's Hospital (Daejeon, Korea) during the 2014-2015 and 2018-2019 influenza seasons were included in this study, and their medical records were retrospectively reviewed. Influenza was diagnosed using a commercial rapid influenza detection test (RIDT) kit (Alere BinaxNOW ${ }^{\circledR}$ Influenza A \& B Card, Abbott, IL, USA). Patients who were positive for both influenza $A$ and $B$ viruses were excluded. Patients in whom a follow-up RIDT was repeated within 2 weeks after the diagnosis of influenza were also excluded. Patients who developed influenza-like illness symptoms $\geq 48$ hours after admission or $<48$ hours after a previous discharge from the hospital were excluded because of the possibility of hospital-acquired infection. For the included patients, demographic data including sex and age, and clinical data including clinical diagnosis, presenting symptoms, chronic underlying diseases, antiviral therapy, and outcomes were collected. Because there were no deaths attributable to influenza during the study period, outcomes were evaluated based on the rates of hospitalization, oxygen supplementation, and mechanical ventilator care. In addition, the development of acute otitis media (AOM) and sinusitis was investigated based on the medical records up to 4 weeks after the diagnosis of influenza. The diagnosis of AOM and sinusitis was made by the clinician's decision based on physical examination and patient's symptoms. The study was conducted in accordance with the Declaration of Helsinki (as revised in 2013). This study was approved by the Institutional Review Board of the Daejeon St. Mary's Hospital with a waiver of acquiring informed consent (approval number: DC19RISI0102).

\section{Definition}

The influenza season was defined from October through May. The included patients were divided into influenza A and $\mathrm{B}$ groups based on their RIDT results. The dominantly circulated subtype of influenza A virus and lineage of influenza $B$ virus were defined based on the results of the annual influenza surveillance of the Korea Disease Control and Prevention Agency (KDCA, Table S1). For influenza A virus, the 2014-2015,2016-2017, and 2017-2018 seasons were categorized as the $\mathrm{A}(\mathrm{H} 3 \mathrm{~N} 2)$ season and the 2015-2016 season was categorized as the A(H1N1) pdm09 season; the 2018-2019 season when the dominantly circulated type comprised less than $80 \%$ of influenza A virus infection cases was not categorized. For influenza B virus, the 2014-2015 and 2017-2018 seasons were categorized as the B/Yamagata season and the 2015-2016 and 2018-2019 
seasons were categorized as the B/Victoria season; the 2016-2017 season when the exact occupying ratios of B/ Yamagata and B/Victoria lineages were not reported was not categorized. Patients who were hospitalized due to influenza-related problems on or within 1 week after the diagnosis of influenza were categorized as the hospitalized group, and the remaining patients were categorized as the non-hospitalized patients. Age groups were divided into 0-2 years (half-dose of trivalent influenza vaccine), 3-6 years (pre-school children), 7-12 years (pre-puberty), and $\geq 13$ years (puberty) with consideration of vaccination effects and biological and social factors. Clinical diagnoses consisted with upper respiratory tract infection (URI), LRI, fever without a focus, and acute gastroenteritis (AGE). URI included rhinitis, pharyngitis, tonsillitis, and croup, and LRI included bronchiolitis, bronchitis, and pneumonia. Among the LRI, suspicious bacterial pneumonia was defined when abnormal breath sounds were heard on auscultation and lobar or segmental consolidations were shown on the chest X-ray. Fever without a focus was defined when there was no abnormality on physical examination in febrile patients without localized symptoms. AGE was defined when gastrointestinal symptoms developed without any respiratory symptoms. If gastrointestinal and respiratory manifestations occurred concurrently, clinical diagnosis was decided based on the respiratory manifestations. Antiviral therapy was defined when 10 or more body weight-adjusted doses of oral oseltamivir or one or more body weightadjusted doses of intravenous peramivir were administered.

\section{Statistical analysis}

The collected demographic and clinical data were compared between the influenza $\mathrm{A}$ and $\mathrm{B}$ groups and between the hospitalized and non-hospitalized groups. The collected data were also compared between the $\mathrm{A}(\mathrm{H} 3 \mathrm{~N} 2)$ and $\mathrm{A}(\mathrm{H} 1 \mathrm{~N} 1) \mathrm{pdm} 09$ seasons of the influenza A group and between the B/Victoria and B/Yamagata seasons of the influenza B group. In each age group, the collected data were also compared between the influenza A and B groups, between the $\mathrm{A}(\mathrm{H} 3 \mathrm{~N} 2)$ and $\mathrm{A}(\mathrm{H} 1 \mathrm{~N} 1) \mathrm{pdm} 09$ seasons of the influenza A group, and between the B/Yamagata and B/ Victoria seasons of the influenza B group. SPSS 21 (IBM Corp., Armonk, NY, USA) was used for statistical analyses. For each comparison between patient groups, continuous and categorical data were compared using Mann-Whitney and Fisher's exact tests, respectively. Statistical significance was defined as a two-sided $\mathrm{P}<0.05$.

\section{Results}

During the study period, RIDTs were performed in 7,807 pediatric patients, and $110(1.4 \%)$ of them were consistent with the exclusion criteria (Figure 1). Of the remaining 7,697 patients, 2,634 (34.2\%) were positive for influenza viruses. Excluding 79 (3.0\%) patients who were positive for both of influenza A and B viruses and three (0.1\%) patients whose medical records were incomplete, 2,552 (96.9\%) patients were eventually included in this study (Figure 1).

\section{Comparison of clinical features and outcomes by influenza virus type, subtype, and lineage}

Of the included 2,552 patients, 1,588 (62.2\%) and 964 (37.8\%) were included in the influenza $\mathrm{A}$ and $\mathrm{B}$ groups, respectively (Table 1). Patients in the influenza $\mathrm{B}$ group were older $(\mathrm{P}<0.001)$ and complained of sore throat more frequently $(\mathrm{P}=0.002)$ than those in the influenza A group. Antiviral therapy was performed in $79.5 \%(n=2,028)$ of the whole study population, and LRI and suspicious bacterial pneumonia were diagnosed in $11.6 \%(n=295)$ and $0.3 \%(n=7)$ of them, respectively. Clinical diagnoses and outcomes were comparable between the patients in the influenza A and B groups (Table 1).

For the influenza A group excluding 298 patients of the 2018-2019 season, patients in the A(H1N1)pdm09 season were younger $(\mathrm{P}=0.003)$ and diagnosed with LRI more frequently $(\mathrm{P}=0.020)$ than those in the $\mathrm{A}(\mathrm{H} 3 \mathrm{~N} 2)$ season (Table 1$)$. Patients in the $\mathrm{A}(\mathrm{H} 3 \mathrm{~N} 2)$ season were more likely to experience myalgia $(\mathrm{P}=0.006)$ and seizures $(\mathrm{P}=0.035)$ than those in the $\mathrm{A}(\mathrm{H} 1 \mathrm{~N} 1) \mathrm{pdm} 09$ season; however, the differences were only $4.1 \%$ and $2.6 \%$, respectively. Between the two influenza A seasons, there were no differences in clinical outcomes (Table 1).

For the influenza B group excluding 46 patients of the 2016-2017 season, patients in the B/Victoria season tended to be younger than those in the B/Yamagata season but without statistical significance (Table 1). There were no differences in clinical features and outcomes between the two seasons except that more patients in the B/Yamagata season complained of myalgia $(\mathrm{P}=0.017)$ than those in the B/Victoria season (Table 1).

\section{Comparison between the non-bospitalized and bospitalized groups}

A total of $571(22.4 \%)$ patients were included in the hospitalized group: 492 (19.3\%) patients were hospitalized 


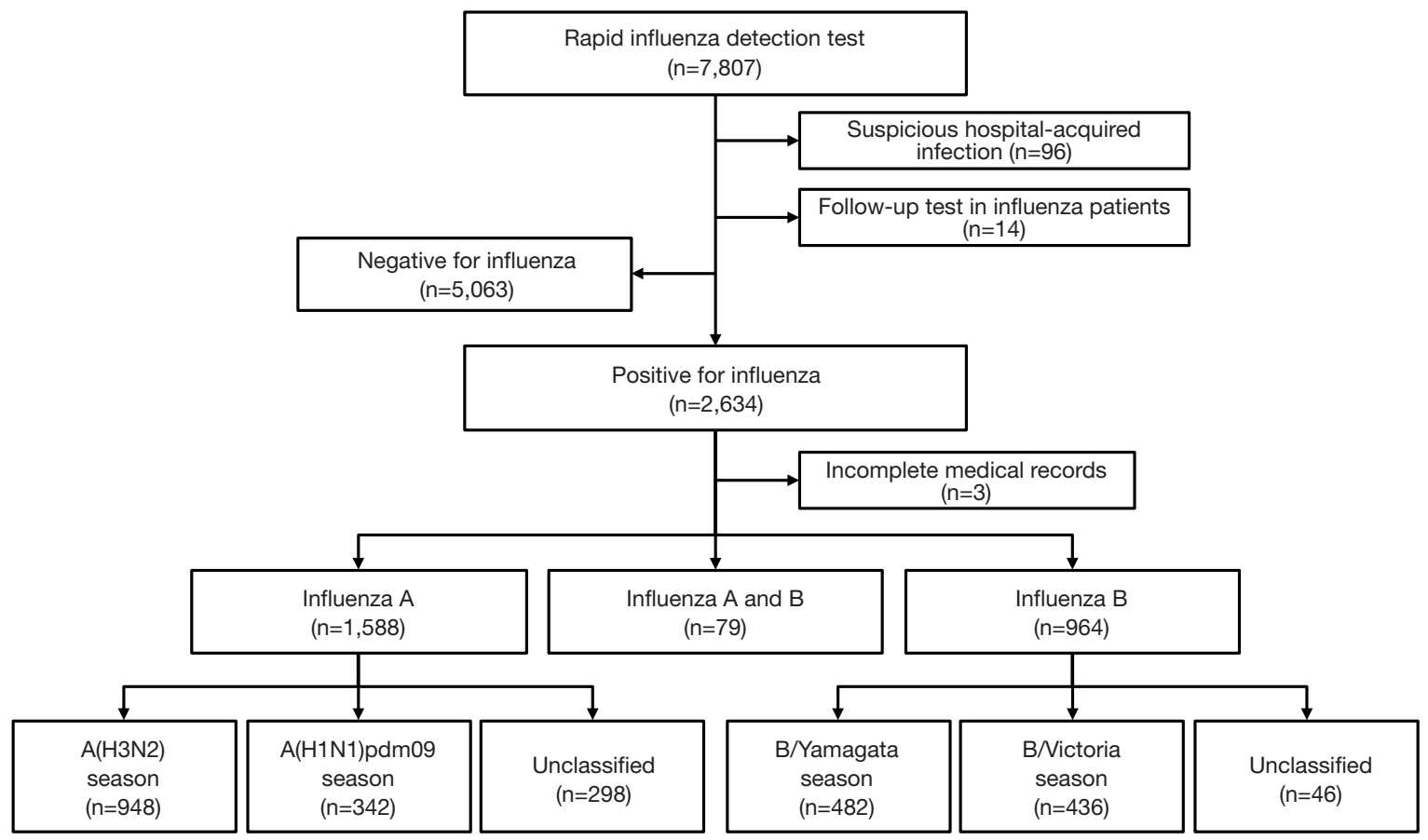

Figure 1 The flowchart for the inclusion and classification of the subjects.

on the day of influenza diagnosis, and $79(3.1 \%)$ patients were hospitalized during follow-up visits after influenza diagnosis. Hospitalized patients were younger $(\mathrm{P}<0.001)$ and diagnosed with LRI more frequently $(\mathrm{P}<0.001)$ than non-hospitalized patients (Table 2). All of the investigated respiratory symptoms except for sore throat occurred more frequently in hospitalized patients than in non-hospitalized patients (Table 2).

For the hospitalized patients, the age distribution between the influenza $\mathrm{A}$ and $\mathrm{B}$ groups was similar (Table 2). Sputum $(\mathrm{P}=0.004)$ and abdominal pain $(\mathrm{P}=0.034)$ were reported more frequently in the influenza $\mathrm{B}$ group than in the influenza A group; however, clinical diagnoses and outcomes were comparable between the influenza $\mathrm{A}$ and $\mathrm{B}$ groups of the hospitalized patients (Table 2).

For the non-hospitalized patients, patients in the influenza $B$ group were older $(\mathrm{P}<0.001)$ and reported sore throat more frequently $(\mathrm{P}=0.004)$ than those in the influenza A group: identical to the results for all included patients (Table 2).

\section{Comparisons of clinical features and outcomes in each age group}

The clinical features and outcomes by the type/subtype/ lineage of influenza virus were compared in each age group because the age distribution was significantly different between the influenza A and B groups (Table 3). In patients aged $0-2$ years, the frequencies of rhinorrhea $(\mathrm{P}=0.042)$, sputum $(\mathrm{P}=0.011)$, LRI $(\mathrm{P}=0.034)$, and hospitalization $(\mathrm{P}=0.001)$ were higher in the influenza $\mathrm{B}$ group than in the influenza $\mathrm{A}$ group (Table 3). In patients aged 3-6 years, there were no significant differences in clinical diagnoses and outcomes between the influenza A and B groups although sore throat was reported more frequently in the influenza $\mathrm{B}$ group than in the influenza A group $(\mathrm{P}=0.003)$. Although no significant differences in demographic and clinical features were identified between the influenza A and B groups in patients aged 7-12 years, LRI was diagnosed more frequently in the influenza $B$ group than in the influenza A group in patients aged 13-19 years $(\mathrm{P}=0.009)$ without significant differences in clinical symptoms and outcomes. There were marginal differences in clinical features between the $\mathrm{A}(\mathrm{H} 3 \mathrm{~N} 2)$ and $\mathrm{A}(\mathrm{H} 1 \mathrm{~N} 1) \mathrm{pdm} 09$ seasons and between the $\mathrm{B} /$ Yamagata and $\mathrm{B} / \mathrm{Victoria}$ seasons in each age group (Tables S2-S5).

\section{Discussion}

In this study, clinical features of pediatric patients diagnosed with influenza during the five influenza seasons were investigated. Clinical features and outcomes showed no 
Table 1 Comparison of clinical features and outcomes by influenza virus type/subtype/lineage

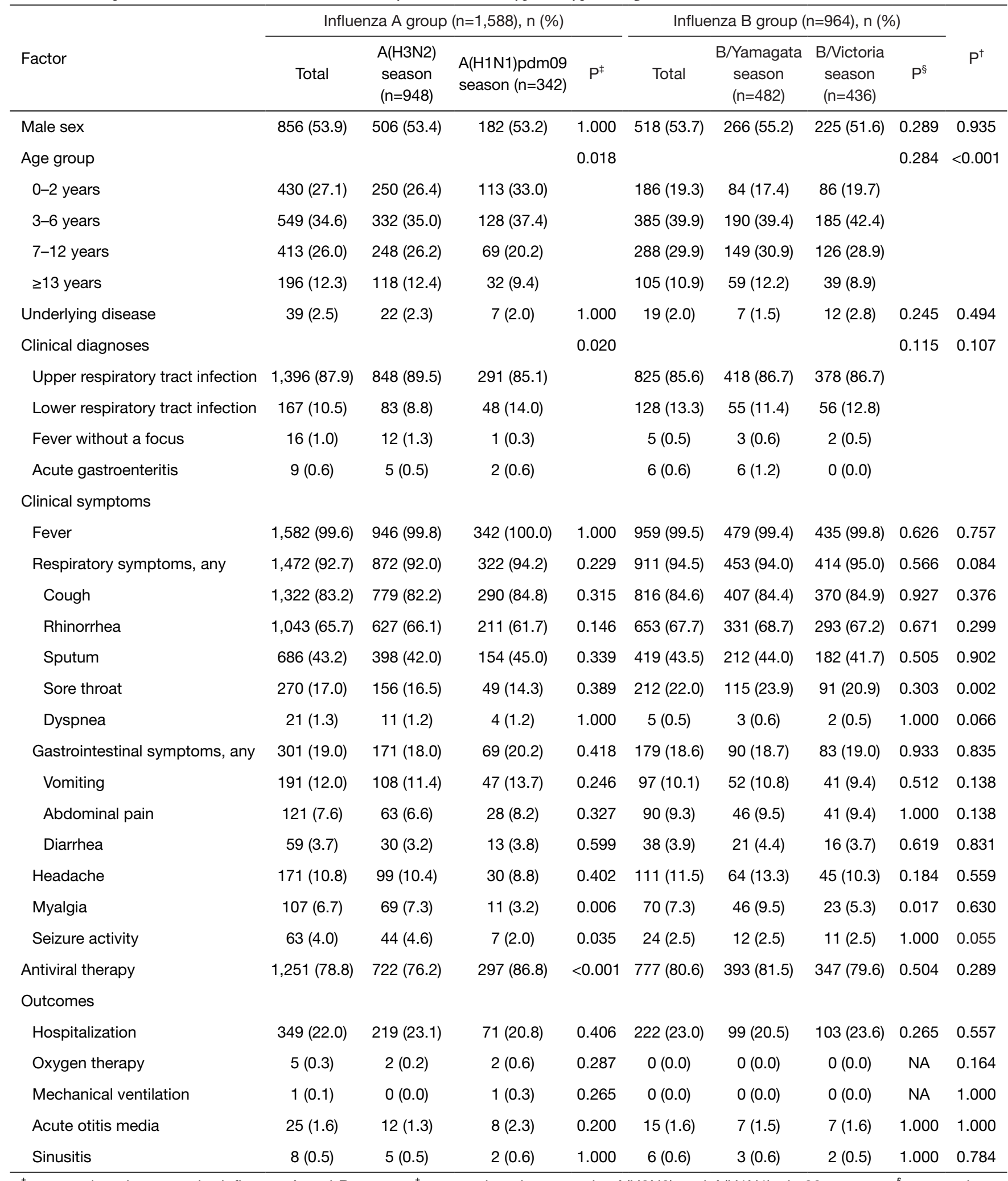

${ }^{\dagger}$, comparison between the influenza $A$ and B groups; ${ }^{\ddagger}$, comparison between the $A(H 3 N 2)$ and $A(H 1 N 1)$ pdm09 seasons; ${ }^{\S}$, comparison between the B/Yamagata and B/Victoria seasons; NA, not applicable. 
Table 2 Comparison of clinical features and outcomes between the non-hospitalized and hospitalized groups

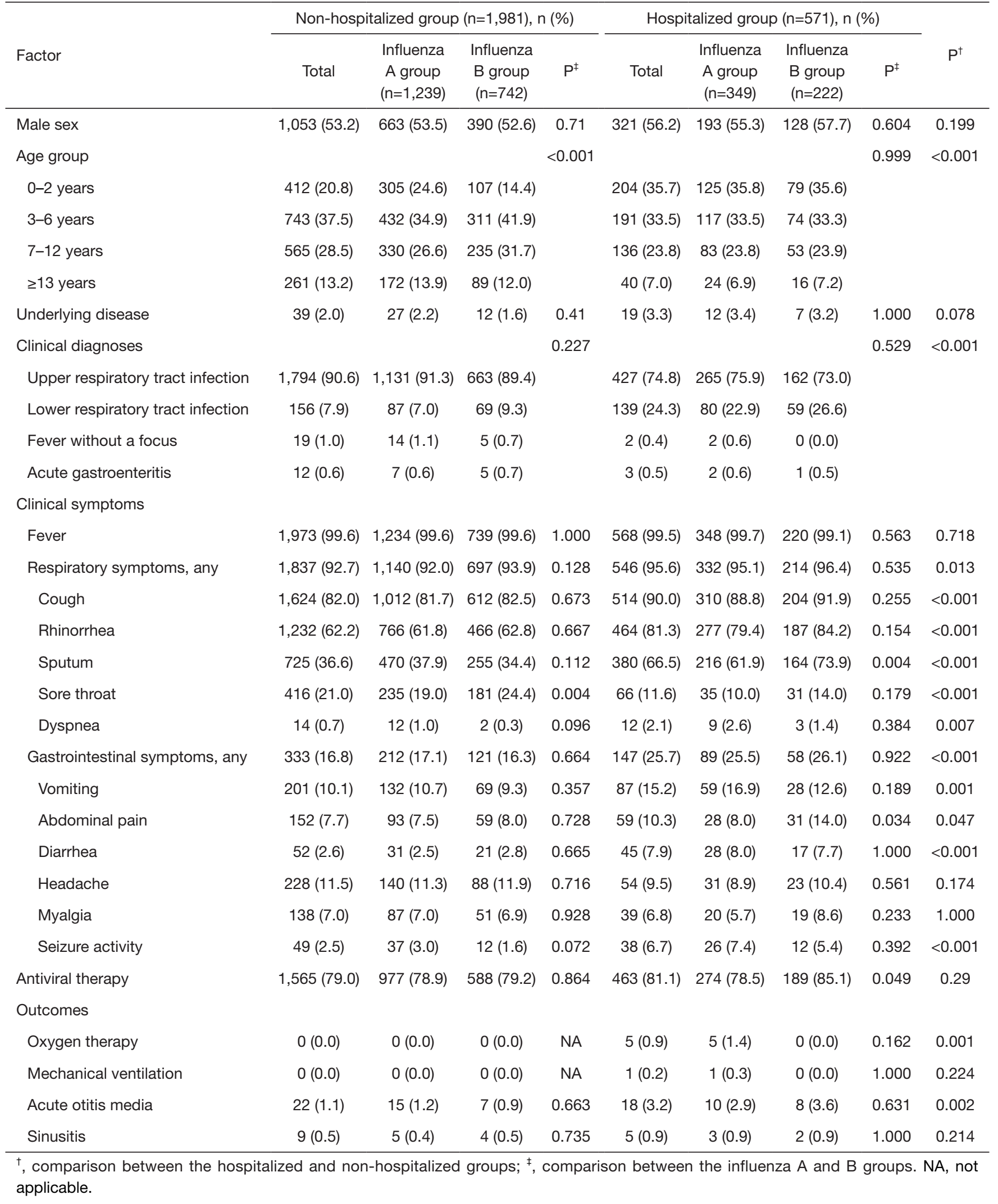


Table 3 Comparison of clinical features and outcomes between the influenza A and B groups in each age group

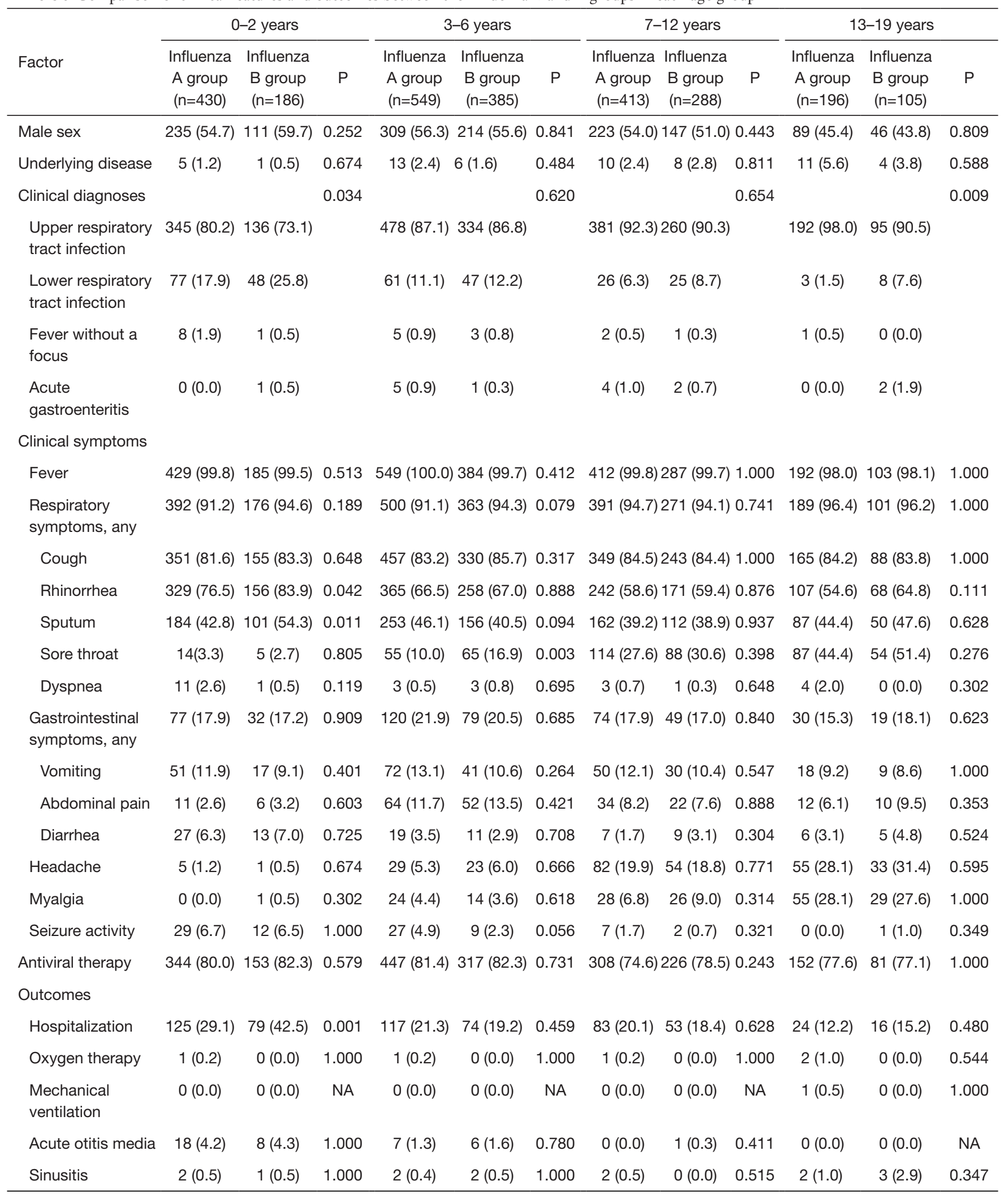

NA, not applicable. 
clinically significant differences according to influenza virus type, subtype, and lineage. However, influenza $B$ virus infection seemed to be more severe than influenza A virus infection in children aged $\leq 2$ years.

The hospitalization rate in this study was $22.4 \%$, which was higher than some previously reported rates of $0.5-3.7 \%$ (9-12), but lower than those of other reported rates of $33-57 \%(12,13)$. Considering no deaths due to influenza, lower rates of secondary bacterial infections, such as AOM (1.6\% vs. $5.6-22.3 \%)$, sinusitis $(0.5 \%$ vs. $2.4-5.9 \%)$, and suspicious bacterial pneumonia $(0.3 \%$ vs. $0.4-6.3 \%)$, and the lower oxygen supplementation rate $(0.2 \%$ vs. $0.8 \%)$ in this study than previously reported rates (10-17), the high hospitalization rate was likely not caused by a truly high severity of influenza in our hospital but by a low medical cost and high access to health care in Korea that was arisen from the National Health Insurance system. The hospitalized patients in this study were younger than the non-hospitalized patients, which was consistent with the increased hospitalization rate due to influenza in children aged $<5$ years (2). Resultantly, the age distribution between the influenza A and B groups of the hospitalized patients was similar, although the age of the influenza A group was younger than that of the influenza B group in the whole study population. Furthermore, respiratory and gastrointestinal symptoms and LRI were more common in the hospitalized patients than in the non-hospitalized patients. Therefore, hospitalized patient-oriented research for the clinical features of influenza may overestimate the symptoms and severity of influenza, although clinical diagnoses and outcomes were similar in the influenza $\mathrm{A}$ and B groups of the hospitalized patients in this study.

Previous studies reported inconsistent but comparable clinical features and outcomes between patients infected with influenza $A$ and $B$ viruses, except for the age distribution $(5,10-16,18)$. The age difference between patients infected with influenza $A$ and $B$ viruses was not same in adults and children. Adult patients infected with influenza A virus were older than those infected with influenza B virus, while, similar to our results, pediatric patients infected with influenza A were younger than those infected with influenza B virus $(12,16,18,19)$. Because there were no clinically significant differences except for age by the type/subtype/lineage of influenza virus in this study, clinical features and outcomes by the type/subtype/ lineage of influenza virus were compared in each age group, showing a few differences in clinical features by the type/ subtype/lineage of influenza virus. In children aged $\leq 2$ years, the influenza $B$ group exhibited higher rates of LRI and hospitalization than the influenza A group. The severity of influenza was higher in patients infected with $\mathrm{A}(\mathrm{H} 3 \mathrm{~N} 2)$ than in those infected with seasonal influenza $\mathrm{A}(\mathrm{H} 1 \mathrm{~N} 1)$ and B viruses in a study performed before the 2009 pandemic (2), which might represent insufficiently accumulated herd immunity against $\mathrm{A}(\mathrm{H} 3 \mathrm{~N} 2)$ in the three decades after the introduction of $\mathrm{A}(\mathrm{H} 3 \mathrm{~N} 2)$ in 1968 (20). Recent studies performed after the 2009 pandemic showed no significant differences in severity between $\mathrm{A}(\mathrm{H} 1 \mathrm{~N} 1) \mathrm{pdm} 09$ and other type/subtype/lineage of influenza viruses $(5,8,10,11,21,22)$. Similarly, in this study, we did not identify a higher severity of $\mathrm{A}(\mathrm{H} 1 \mathrm{~N} 1)$ pdm09 infection than other influenza virus infections, although only about 10 years have passed since the 2009 pandemic. The relatively high vaccination rate against influenza in Korean children (about 50\%), especially in those aged $<5$ years (about $80 \%$ ), through the 2010 s may contribute to maintaining sufficient herd immunity against $\mathrm{A}(\mathrm{H} 1 \mathrm{~N} 1)$ pdm09 and reducing the severity of $\mathrm{A}(\mathrm{H} 1 \mathrm{N1}) \mathrm{pdm} 09$ infection (23). The combination of a high vaccination rate and high rate $(79.5 \%)$ of antiviral therapy in this study might further decrease the severity of influenza A virus infection. Because quadrivalent influenza vaccines for children aged $\leq 2$ years were approved in Korea in 2018 , almost none of the patients aged $\leq 2$ years included in this study have been exposed to both of the lineages of influenza $B$ virus by vaccination and natural infection. Lower levels of protective immunity against influenza B virus compared with influenza $\mathrm{A}$ virus might cause the different frequencies of LRI and hospitalization between influenza $\mathrm{A}$ and $\mathrm{B}$ groups in children aged $\leq 2$ years of age. Expanded use of quadrivalent influenza vaccines in infants and young children could affect the clinical features and severity of influenza $B$ virus infection in the future. LRI was also diagnosed more frequently in the influenza B group than in the influenza A group in patients aged 13-19 years. However, clinical outcomes including hospitalization rate were not significantly different between the influenza A and B groups, and the frequency of LRI in this age group was lower than that in other age groups. There has been no report that compared clinical features of influenza $\mathrm{A}$ and $\mathrm{B}$ virus infection exclusively in adolescents. This should be further explored. Although $\mathrm{A}(\mathrm{H} 3 \mathrm{~N} 2)$ did not cause more severe infection than $\mathrm{A}(\mathrm{H} 1 \mathrm{~N} 1) \mathrm{pdm} 09$, recently, $\mathrm{A}(\mathrm{H} 3 \mathrm{~N} 2)$ has circulated dominantly during more influenza seasons than $\mathrm{A}(\mathrm{H} 1 \mathrm{~N} 1)$ pdm09. Efforts for improving the immunogenicity against $\mathrm{A}(\mathrm{H} 3 \mathrm{~N} 2)$ in influenza vaccines should be conducted along with the use of quadrivalent 
influenza vaccines.

This study had some limitations. The retrospective nature of this study made it impossible to determine the exact time of symptom onset and termination and influenza vaccination rates for the included patients, and to avoid selection bias. The low rates of AOM and sinusitis might be due to the lack of unified diagnostic criteria for AOM and sinusitis applied in this study, and therefore, some cases with $\mathrm{AOM}$ and sinusitis might be missed. However, the low rates could represent truly low rates of secondary bacterial complications in patients receiving antiviral therapy at a high rate. Because the subtype and lineage of the influenza viruses were determined based on the surveillance data of the KDCA rather than individualized tests in each patient, clinical features of each influenza season might be complicated by the other type or lineage of virus. To overcome this limitation, only the influenza seasons when the dominantly circulated subtype or lineage occupied $\geq 80 \%$ of the identified influenza type were included in the analysis. However, regional differences in the distribution of subtype and lineage of circulated influenza virus might be present even in the same country. Therefore, each hospital should collect its own data on epidemiological and clinical characteristics of influenza patients based on the regional and national epidemiological changes in influenza virus infection. The application of a polymerase chain reaction (PCR) test with a higher sensitivity compared to an RIDT may affect clinical features or outcomes of influenza. However, the PCR test tends to detect a lower viral load compared with RIDT, and therefore, influenza patients with milder symptoms can be more easily identified with the use of a PCR test. Although other co-infected respiratory pathogens might modify clinical features and outcomes of influenza, a multiplex PCR test identifying multiple respiratory pathogens simultaneously was not applied in most of the included patients. A prospective study adjusted for above-mentioned limitations should be adequate for determining clinical features and outcomes of influenza caused by recently circulating viruses.

\section{Conclusions}

There were no clinically significant differences by the type/ subtype/lineage among patients infected with four recently circulating influenza viruses. In children aged $\leq 2$ years, influenza $B$ virus infection seemed to be more severe than influenza A virus infection. Considering the recent approval of quadrivalent influenza vaccines for children aged between 6 and 36 months and their expected expanded use in infants and young children, repeat studies on the clinical differences of influenza by the virus type/subtype/lineage in each age group should be planned.

\section{Acknowledgments}

Funding: None.

\section{Footnote}

Reporting Checklist: The authors have completed the STROBE reporting checklist. Available at http://dx.doi. org/10.21037/tp-20-196

Data Sharing Statement: Available at http://dx.doi. org/10.21037/tp-20-196

Conflicts of Interest: All authors have completed the ICMJE uniform disclosure form (available at http://dx.doi. org/10.21037/tp-20-196). The authors have no conflicts of interest to declare.

Ethical Statement: The authors are accountable for all aspects of the work in ensuring that questions related to the accuracy or integrity of any part of the work are appropriately investigated and resolved. The study was conducted in accordance with the Declaration of Helsinki (as revised in 2013) and the Harmonized Tripartite Guideline for Good Clinical Practice from the International Conference on Harmonization. This study was reviewed and approved by the Institutional Review Board of the Daejeon St. Mary's Hospital with a waiver of acquiring informed consent (approval number: DC19RISI0102).

Open Access Statement: This is an Open Access article distributed in accordance with the Creative Commons Attribution-NonCommercial-NoDerivs 4.0 International License (CC BY-NC-ND 4.0), which permits the noncommercial replication and distribution of the article with the strict proviso that no changes or edits are made and the original work is properly cited (including links to both the formal publication through the relevant DOI and the license). See: https://creativecommons.org/licenses/by-nc-nd/4.0/.

\section{References}

1. Influenza(Seasonal) [Internet]. Geneva: World Health 
Organization; c2020 [cited 2020 Jul 15]. Available online: https://www.who.int/news-room/fact-sheets/detail/ influenza-(seasonal).

2. Thompson WW, Shay DK, Weintraub E, et al. Influenzaassociated hospitalizations in the United States. JAMA 2004;292:1333-40.

3. Shang M, Balnton L, Brammer L, et al. Influenzaassociated pediatric deaths in the United States, 2010 2016. Pediatrics 2018;141:e20172918.

4. Peters TR, Snively BM, Suerken CK, et al. Relative timing of influenza disease by age group. Vaccine 2014;32:6451-6.

5. Caini S, Kroneman M, Wiegers T, et al. Clinical characteristics and severity of influenza infections by virus type, subtype, and lineage: A systematic literature review. Influenza Other Respir Viruses 2018;12:780-92.

6. Ambrose CS, Levin MJ. The rationale for quadrivalent influenza vaccines. Hum Vaccin Immunother 2012;8:81-8.

7. Chi CY, Wang SH, Lin CC, et al. Clinical features of children infected with different strains of influenza B in southern Taiwan. Pediatr Infect Dis J 2008;27:640-5.

8. Mancinelli L, Onori M, Concato C, et al. Clinical features of children hospitalized with influenza $\mathrm{A}$ and $\mathrm{B}$ infections during the 2012-2013 influenza season in Italy. BMC Infect Dis 2016;16:6.

9. Belongia EA, Irving SA, Waring SC, et al. Clinical characteristics and 30-day outcomes for influenza A 2009 (H1N1), 2008-2009 (H1N1), and 2007-2008 (H3N2) infections. JAMA 2010;304:1091-8.

10. Streng A, Prifert C, Weissbrich B, et al. Subtypespecific clinical presentation, medical treatment and family impact of influenza in children 1-5 years of age treated in outpatient practices in Germany during three postpandemic years, 2013-2015. Pediatr Infect Dis J 2018;37:861-7.

11. Hawkes M, Schuh S, Ipp M, et al. Natural history of pandemic H1N1 2009 influenza infection in healthy pediatric outpatients. Acad Pediatr 2011;11:66-74.

12. Esposito S, Cantarutti L, Molteni CG, et al. Clinical manifestations and socio-economic impact of influenza

Cite this article as: Han SB, Rhim JW, Kang JH, Lee KY. Clinical features and outcomes of influenza by virus type/ subtype/lineage in pediatric patients. Transl Pediatr 2021;10(1):54-63. doi: 10.21037/tp-20-196 among healthy children in the community. J Infect 2011;62:379-87.

13. Peltola V, Ziegler T, Ruuskanen O. Influenza A and B virus infections in children. Clin Infect Dis 2003;36:299-305.

14. Mosnier A, Caini S, Daviaud I, et al. Clinical characteristics are similar across type A and B influenza virus infections. PLoS One 2015;10:e0136186.

15. Silvennoinen H, Huusko T, Vuorinen T, et al. Comparative burden of influenza A/H1N1, A/H3N2 and $\mathrm{B}$ infections in children treated as outpatients. Pediatr Infect Dis J 2015;34:1081-5.

16. Bennet R, Hamrin J, Wirgart BZ, et al. Influenza epidemiology among hospitalized children in Stockholm, Sweden 1998-2014. Vaccine 2016;34:3298-302.

17. Heikkinen T, Silvennoinen H, Peltola V, et al. Burden of influenza in children in the community. J Infect Dis 2004;190:1369-73.

18. Hite LK, Glezen WP, Demmler GJ, et al. Medically attended pediatric influenza during the resurgence of the Victoria lineage of influenza B virus. Int J Infect Dis 2007;11:40-7.

19. Hinds AM, Bozat-Emre S, Van Caeseele P, et al. Comparison of the epidemiology of laboratory-confirmed influenza A and influenza B cases in Manitoba, Canada. BMC Public Health 2015;15:35.

20. Elliot AJ, Fleming DM. Surveillance of influenza-like illness in England and Wales during 1966-2006. Euro Surveill 2006;11:249-50.

21. Heininger U, Baer G, Ryser AJ, et al. Comparative analysis of clinical characteristics of pandemic influenza a/h1n1 and seasonal influenza a infections in hospitalized children. Pediatr Infect Dis J 2013;32:293-6.

22. Zhang T, Zhu Q, Zhang X, et al. The clinical characteristics and direct medical cost of influenza in hospitalized children: a five-year retrospective study in Suzhou, China. PLoS One 2012;7:e44391.

23. KOrean Statistical Information Service [Internet]. Seoul, Korea: Statistics Korea; c2020 [cited 2020 Jul 15]. Available online: http://kosis.kr/index/index.do 
Supplementary

Table S1 Dominantly circulated influenza viruses based on the Korea Disease Control and Prevention Agency surveillance reports during the study period in Korea

\begin{tabular}{|c|c|c|c|c|c|}
\hline Influenza season & 2014-2015 season & 2015-2016 season & 2016-2017 season & 2017-2018 season & 2018-2019 season \\
\hline Influenza A (H3N2) & $83 \%$ & $10 \%$ & $99 \%$ & $85 \%$ & $67 \%$ \\
\hline Influenza A (H1N1)pdm09 & $17 \%$ & $90 \%$ & $1 \%$ & $15 \%$ & $33 \%$ \\
\hline Influenza B virus & $37 \%$ & $51 \%$ & $27 \%$ & $55 \%$ & $37 \%$ \\
\hline Influenza B/Victoria & $0 \%$ & $97 \%$ & Unknown & $1 \%$ & $99 \%$ \\
\hline
\end{tabular}


Table S2 Comparison of clinical features and outcomes by the influenza virus type/subtype/lineage in patients aged 0-2 years

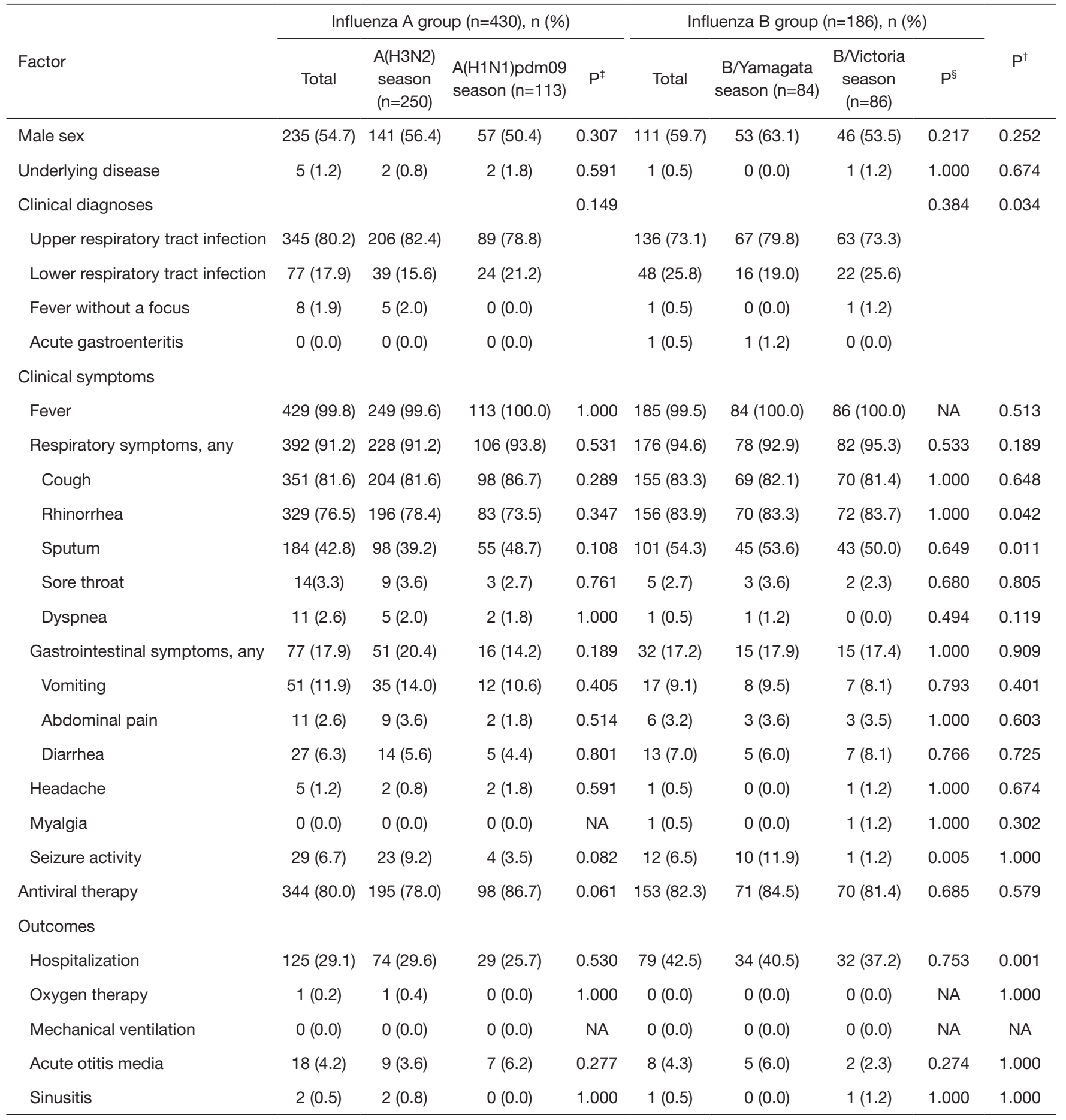

${ }^{\dagger}$, Comparison between the influenza A and B groups; ${ }^{\ddagger}$, Comparison between the $\mathrm{A}(\mathrm{H} 3 \mathrm{~N} 2)$ and $\mathrm{A}(\mathrm{H} 1 \mathrm{~N} 1)$ pdm09 seasons; ${ }^{\S}$, Comparison between the B/Yamagata and B/Victoria seasons; NA, not applicable. 
Table S3 Comparison of clinical features and outcomes by the influenza virus type/subtype/lineage in patients aged 3-6 years

\begin{tabular}{|c|c|c|c|c|c|c|c|c|c|}
\hline Factor & \multicolumn{4}{|c|}{ Influenza A group ( $\mathrm{n}=549), \mathrm{n}(\%)$} & \multicolumn{4}{|c|}{ 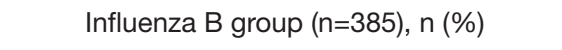 } & $\mathrm{P}^{\dagger}$ \\
\hline Male sex & $309(56.3)$ & $185(55.7)$ & $73(57.0)$ & 0.834 & $214(55.6)$ & $109(57.4)$ & $100(54.1)$ & 0.534 & 0.841 \\
\hline Underlying disease & $13(2.4)$ & $8(2.4)$ & $1(0.8)$ & 0.455 & $6(1.6)$ & $4(2.1)$ & $2(1.1)$ & 0.685 & 0.484 \\
\hline Upper respiratory tract infection & $478(87.1)$ & $298(89.8)$ & $108(84.4)$ & & $334(86.8)$ & $164(86.3)$ & $164(88.6)$ & & \\
\hline Lower respiratory tract infection & $61(11.1)$ & $26(7.8)$ & $18(14.1)$ & & $47(12.2)$ & $22(11.6)$ & $21(11.4)$ & & \\
\hline Fever without a focus & $5(0.9)$ & $5(1.5)$ & $0(0.0)$ & & $3(0.8)$ & $3(1.6)$ & $0(0.0)$ & & \\
\hline Acute gastroenteritis & $5(0.9)$ & $3(0.9)$ & $2(1.6)$ & & $1(0.3)$ & $1(0.5)$ & $0(0.0)$ & & \\
\hline Respiratory symptoms, any & $500(91.1)$ & $297(89.5)$ & $118(92.2)$ & 0.484 & $363(94.3)$ & $178(93.7)$ & $176(95.1)$ & 0.655 & 0.079 \\
\hline Cough & 457 (83.2) & $271(81.6)$ & $108(84.4)$ & 0.585 & $330(85.7)$ & $160(84.2)$ & $161(87.0)$ & 0.465 & 0.317 \\
\hline Rhinorrhea & $365(66.5)$ & $215(64.8)$ & $79(61.7)$ & 0.588 & $258(67.0)$ & $128(67.4)$ & $123(66.5)$ & 0.913 & 0.888 \\
\hline Sputum & $253(46.1)$ & $155(46.7)$ & $57(44.5)$ & 0.754 & $156(40.5)$ & $81(42.6)$ & $71(38.4)$ & 0.462 & 0.094 \\
\hline Sore throat & $55(10.0)$ & $37(11.1)$ & $11(8.6)$ & 0.498 & 65 (16.9) & $29(15.3)$ & $36(19.5)$ & 0.34 & 0.003 \\
\hline Dyspnea & $3(0.5)$ & $1(0.3)$ & $1(0.8)$ & 0.480 & $3(0.8)$ & $2(1.1)$ & $1(0.5)$ & 1.000 & 0.695 \\
\hline Gastrointestinal symptoms, any & $120(21.9)$ & $64(19.3)$ & $31(24.2)$ & 0.249 & $79(20.5)$ & $38(20.0)$ & $39(21.1)$ & 0.800 & 0.685 \\
\hline Seizure activity & $27(4.9)$ & $16(4.8)$ & $3(2.3)$ & 0.302 & $9(2.3)$ & $1(0.5)$ & $8(4.3)$ & 0.019 & 0.056 \\
\hline Antiviral therapy & $447(81.4)$ & $255(76.8)$ & $114(89.1)$ & 0.003 & 317 (82.3) & $159(83.7)$ & $151(81.6)$ & 0.683 & 0.731 \\
\hline \multicolumn{10}{|l|}{ Outcomes } \\
\hline Hospitalization & 117 (21.3) & $74(22.3)$ & 25 (19.5) & 0.613 & $74(19.2)$ & $33(17.4)$ & $38(20.5)$ & 0.510 & 0.459 \\
\hline Oxygen therapy & $1(0.2)$ & $0(0.0)$ & $1(0.8)$ & 0.278 & $0(0.0)$ & $0(0.0)$ & $0(0.0)$ & NA & 1.000 \\
\hline Mechanical ventilation & $0(0.0)$ & $0(0.0)$ & $0(0.0)$ & NA & $0(0.0)$ & $0(0.0)$ & $0(0.0)$ & NA & NA \\
\hline Acute otitis media & $7(1.3)$ & $3(0.9)$ & $1(0.8)$ & 1.000 & $6(1.6)$ & $1(0.5)$ & $5(2.7)$ & 0.118 & 0.78 \\
\hline Sinusitis & $2(0.4)$ & $0(0.0)$ & $1(0.8)$ & 0.278 & $2(0.5)$ & $1(0.5)$ & $1(0.5)$ & 1.000 & 1.000 \\
\hline
\end{tabular}

${ }^{\dagger}$, comparison between the influenza A and B groups; ${ }^{\ddagger}$, Comparison between the $A(H 3 N 2)$ and $A(H 1 N 1)$ pdm09 seasons; ${ }^{\S}$, comparison between the B/Yamagata and B/Victoria seasons. NA, not applicable. 
Table S4 Comparison of clinical features and outcomes by the influenza virus type/subtype/lineage in patients aged 7-12 years

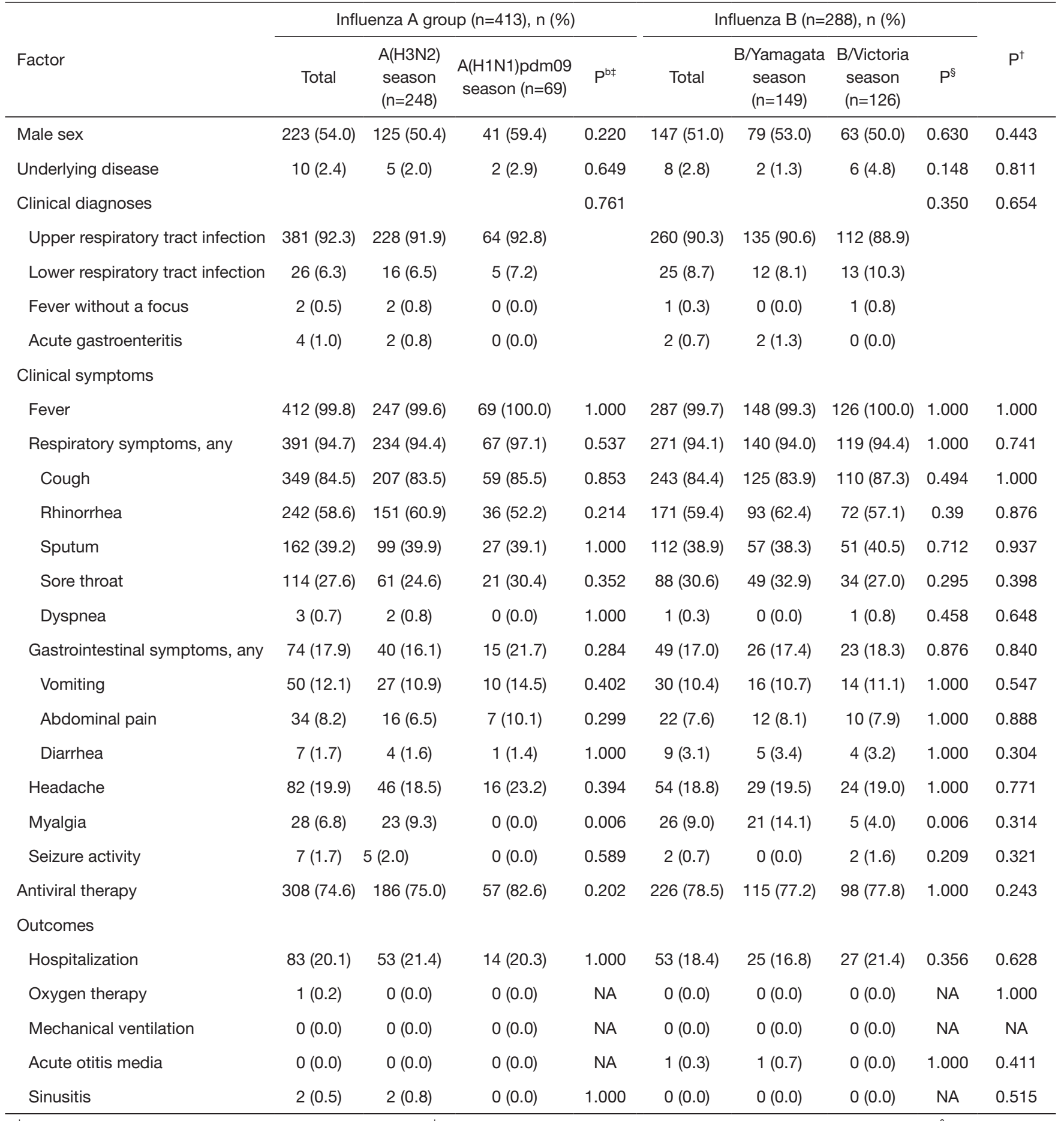

${ }^{\dagger}$, comparison between the influenza A and B groups; ${ }^{\ddagger}$, comparison between the $\mathrm{A}(\mathrm{H} 3 \mathrm{~N} 2)$ and $\mathrm{A}(\mathrm{H} 1 \mathrm{~N} 1)$ pdm09 seasons; ${ }^{\S}$, comparison between the B/Yamagata and B/Victoria seasons. NA, not applicable. 
Table S5 Comparison of clinical features and outcomes by the influenza virus type/subtype/lineage in patients aged 13-19 years

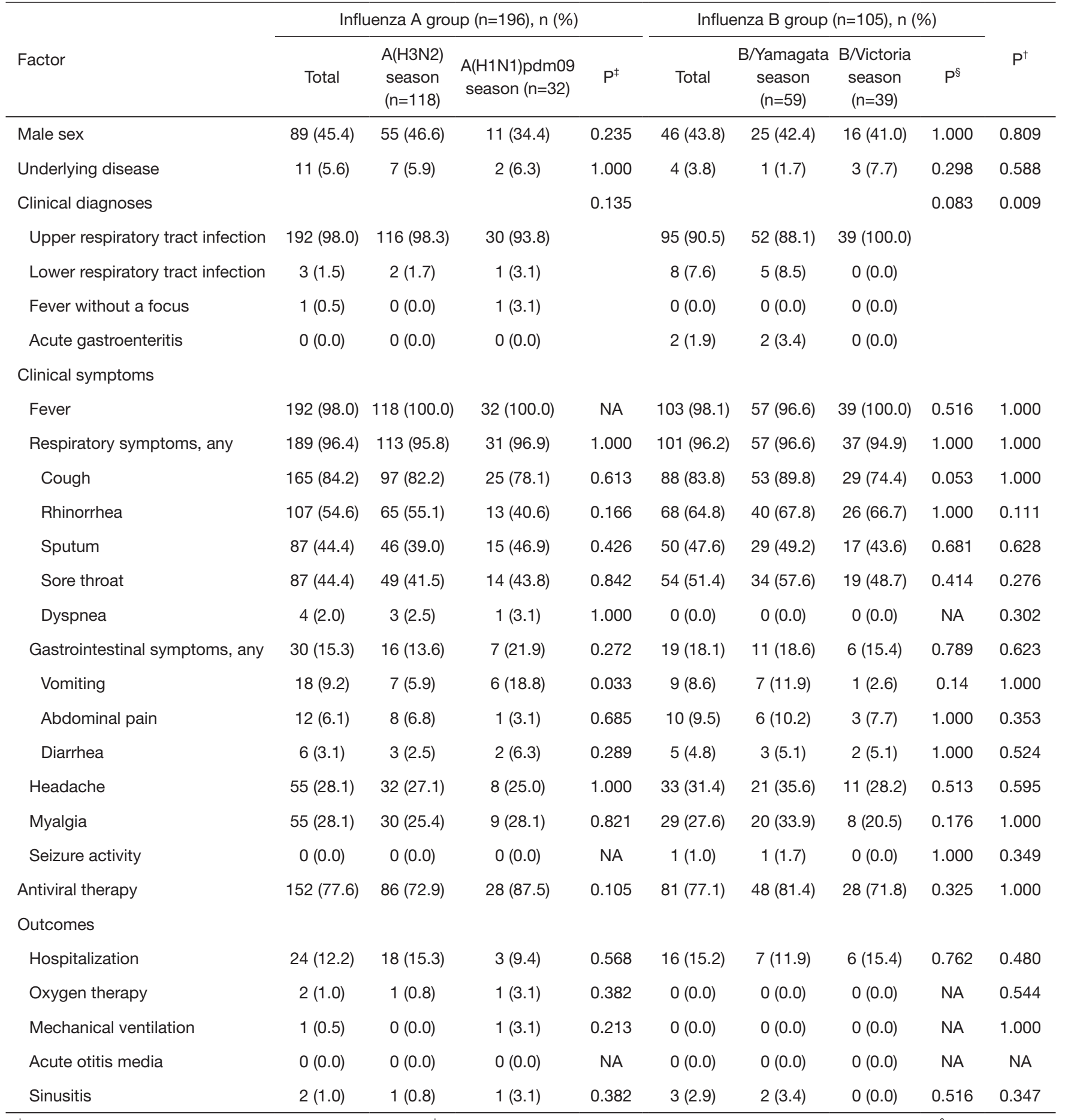

${ }^{\dagger}$, comparison between the influenza A and B groups; ${ }^{\ddagger}$, comparison between the $\mathrm{A}(\mathrm{H} 3 \mathrm{~N} 2)$ and $\mathrm{A}(\mathrm{H} 1 \mathrm{~N} 1)$ pdm09 seasons; ${ }^{\S}$, comparison between the B/Yamagata and B/Victoria seasons. NA, not applicable. 